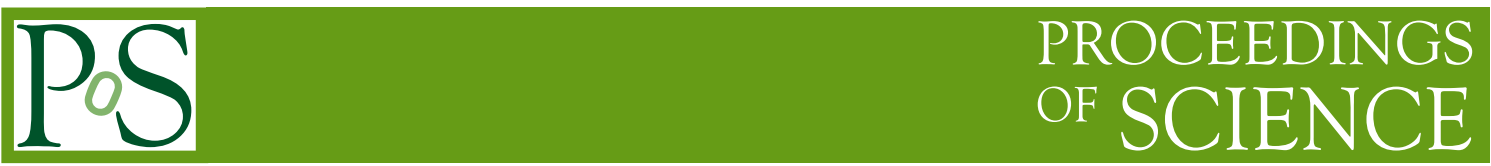

\title{
Boosted Top quark polarization
}

\author{
Aravind H. Vijay ${ }^{* \dagger}$ \\ Tata Institute of Fundamental Research, Mumbai \\ E-mail: aravindhv10@gmail.com
}

In top quark production, the polarization of top quarks, decided by the chiral structure of couplings, is likely to be modified in the presence of any new physics contribution to the production. Hence it is a good discriminator for those new physics models wherein the couplings have a chiral structure different than that in the Standard Model (SM). In this note we construct probes of the polarization of a top quark decaying hadronically, using easily accessible kinematic variables such as the energy fraction or angular correlations of the decay products. Tagging the boosted top quark using jet sub structure technique, we study robustness of these observables for a benchmark process, $W^{\prime} \rightarrow t b$. We demonstrate that the energy fraction of b-jet in the laboratory frame and a new angular variable, constructed by us in the top rest frame, are both very powerful tools to discriminate between the left and right polarized top quarks. Based on the polarization sensitive angular variables, we construct asymmetries which reflect the polarization. We study the efficacy of these variables for a new physics processes which give rise to boosted top quarks from the decay of the top squark in the context of supersymmetry searches. Remarkably, it is found that the asymmetry can vary over a wide range about $+20 \%$ to $-20 \%$. The dependence of asymmetry on top quark couplings of the new particles present in these models beyond the SM (BSM) is also investigated in detail.

European Physical Society Conference on High Energy Physics - EPS-HEP2019 -

10-17 July, 2019

Ghent, Belgium

\footnotetext{
* Speaker.

${ }^{\dagger}$ Based on work in collaboration with Monoranjan Guchait, Jayita Lahiri, Rohini Godbole, Charanjit K. Khosa, Seema Sharma [1].
} 


\section{Introduction}

The study of top quark polarization is an extremely interesting and relevant problem. The Top quark decays before it can hadronize, hence it's polarization state is protected from effects of softQCD, this imprint is visible in the kinematics of it's decay products. In the standard model (SM), it can be used to probe anomalous $W-t-b$ couplings given by Eq. 1.1.

$$
\Gamma^{\mu}=\begin{aligned}
& \frac{-i g}{\sqrt{2}}\left[\gamma^{\mu}\left\{\left(1+f_{1 L}\right) \mathbf{P}_{L}+f_{1 R} \mathbf{P}_{R}\right\}\right] \\
& -\frac{g \sigma^{\mu \nu}}{\sqrt{2} m_{W}}\left[\left(p_{t}-p_{b}\right)_{v}\left(f_{2 L} \mathbf{P}_{L}+f_{2 R} \mathbf{P}_{R}\right)\right]
\end{aligned}
$$

Study of polarization also helps us to determine the nature of couplings in the underlying model responsible for it's production and decay. For instance, since it is the heaviest known particle, it has one of the largest couplings with the Higgs sectors of many models beyond SM (BSM), thereby providing a valuable tool to probe the Higgs sectors of these models. Our main goal in this study is to construct good observables which are sensitive to the polarization of boosted hadronically decaying tops (tagged using the HEPTopTagger algorithm [2, 3]) and benchmark them in a toy and realistic BSM scenario.

\section{Polarized top quark decays}

The kinematics of top quark decay is given by Eq. 2.1

$$
\frac{1}{\Gamma} \frac{d \Gamma}{d \cos \theta_{f}}=\frac{1}{2}\left(1+\mathscr{P}_{0} \kappa_{f} \cos \theta_{f}\right),
$$

where $\theta_{f}$ is the angle between top spin and decay product momentum, $\mathscr{P}_{0}$ is the polarization of the produced top, $\kappa_{f}$ is the spin analyzing power [4] with $-\kappa_{W^{ \pm}}=\kappa_{b} \approx-0.4, \kappa_{\bar{d}}=1$ and $\kappa_{u} \approx-0.3$. From this we notice that $\bar{d}$ (or the lepton) has the highest spin analyzing power but it's identity is lost in a hadronic decay, it can be shown that $\bar{d}$ quark tends to forms the smallest invariant mass with $b$ quark, this feature can be exploited to construct strong polarization discrimination observables.

$$
\cos \theta^{*} \equiv \frac{\overrightarrow{t_{j}} \cdot \overrightarrow{j_{1}^{\prime}}}{\left|\vec{t}_{j}\right|\left|\vec{j}_{1}^{\prime}\right|}
$$

where, $\vec{t}_{j}$ is the momentum of the top tagged jet in the lab frame, $j_{1}$ is the sub jet which forms the least invariant mass with the $b$-like subjet and $\vec{j}_{1}^{\prime}$ is it's momentum in the top rest frame.

\section{Boosted top polarization}

For the purpose of benchmarking, we consider the simple process:

$$
p p \rightarrow W^{\prime} \rightarrow t b
$$

in the $W^{\prime}$ effective model given by the lagrangian $[5,6]$ :

$$
\mathscr{L} \supset \frac{V_{i j}}{2 \sqrt{2}} \bar{f}_{i} \gamma^{\mu}\left(g_{R}\left(1+\gamma_{5}\right)+g_{L}\left(1-\gamma_{5}\right)\right) W_{\mu}^{\prime} f_{j}+\text { h.c. },
$$


the events are generated using Pythia8 [7].

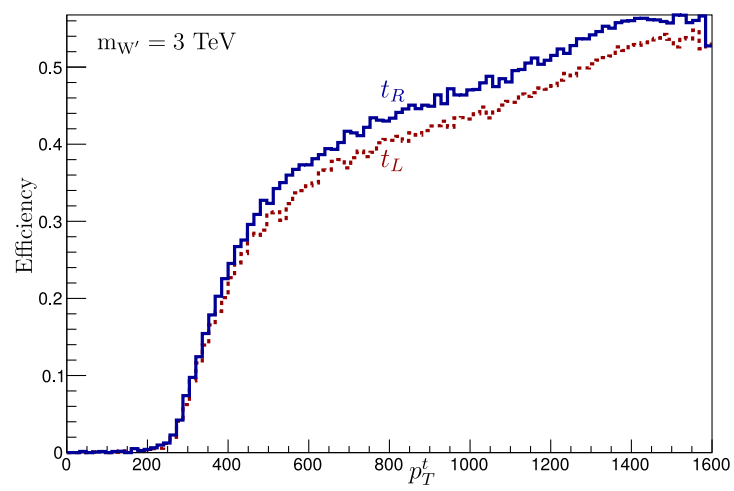

Figure 1: Top tagging efficiency for left $\left(t_{L}\right)$ and right chiral $\left(t_{R}\right)$ top quark for the process $p p \rightarrow W^{\prime} \rightarrow t b$ with $m_{W^{\prime}}=3 \mathrm{TeV}$.
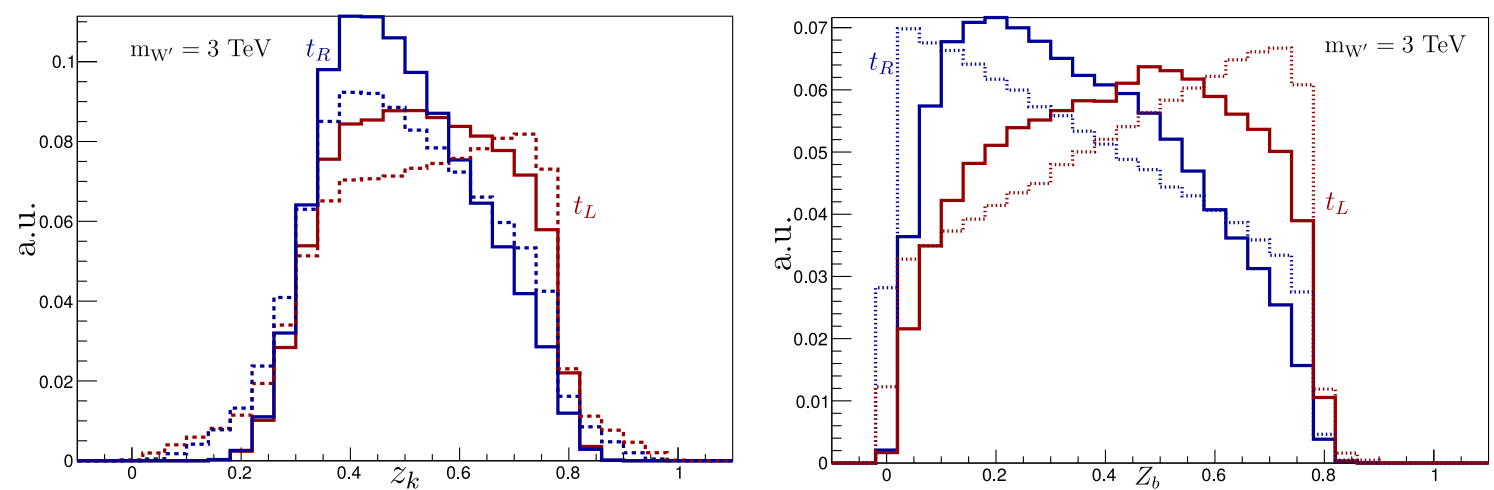

The energy fraction $z_{k}$ from Ref. [8]

Energy fraction of $b$-like subjet $\left(E_{b} / E_{t}\right)$

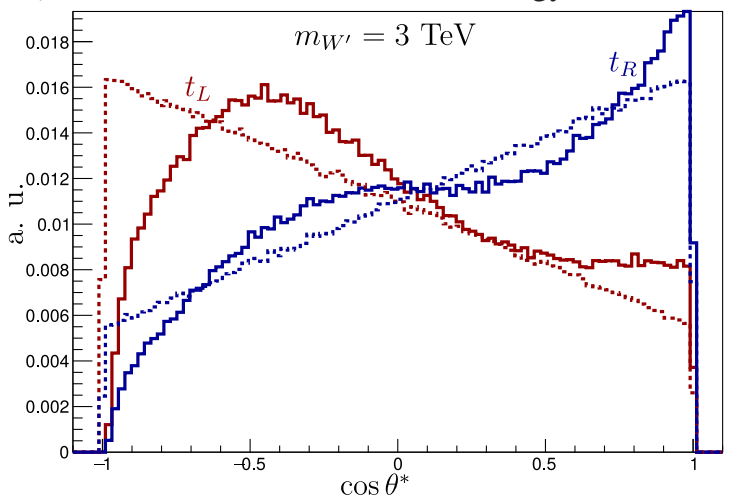

The $\cos \theta^{*}$ distribution (Eq. 2.2)

Figure 2: Distributions of the various observables for left (red) and right (blue) handed top quark for the benchmark process Eq. 3.1 with $m_{W^{\prime}}=3 \mathrm{TeV}$. The dashed lines correspond to the observable at the partonic level while solid lines correspond to the observable after reconstruction and top tagging using HEPTopTagger2.

It turns out, that due to the difference in the kinematics between the left and right handed tops, 
there is slight difference in the top tagging efficiency. This difference can be seen in Fig. 1.

In addition to the observable described in Eq. 2.2, we have also considered the energy fraction observable described in [8] and also the energy fraction of the $b$-like subjet (evaluated by the $W^{ \pm}$mass reconstruction condition) inside the top fatjet $\left(E_{b} / E_{t}\right)$, these distributions are presented in Fig. 2. The distinct characteristic of $\cos \theta^{\star}$ is exploited in constructing the event asymmetry defined as,

$$
A_{\theta^{*}} \equiv \frac{N_{\cos \theta^{*}>0}-N_{\cos \theta^{*}<0}}{N_{\cos \theta^{*}>0}+N_{\cos \theta^{*}<0}}
$$

where $N$ represents the number of events for the given condition of $\cos \theta^{\star}$, either positive or negative, this distribution is presented in Fig. 3.

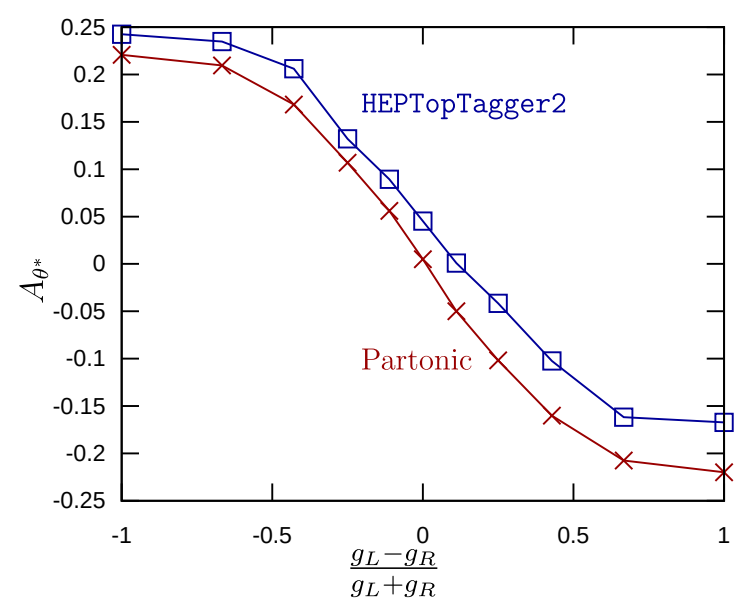

Figure 3: The asymmetry (Eq. 3.3) for parton level and tagged tops for $m_{W^{\prime}}=3 \mathrm{TeV}$.

\section{Top polarization in stop decay}

Now, we consider the pair production and decay of the stop $\left(\tilde{t}_{1}\right)$ squark (in MSSM) to the top and $\operatorname{LSP}\left(\tilde{\chi}_{1}^{0}\right)$ [Eq. 4.1].

$$
p p \rightarrow \tilde{t}_{1} \overline{\tilde{t}}_{1} \rightarrow t \bar{t} \tilde{\chi}_{1}^{0} \tilde{\chi}_{1}^{0}
$$

The compositions of $\tilde{t}_{1}$ and $\tilde{\chi}_{1}^{0}$ are given by:

$$
\begin{aligned}
\tilde{\chi}_{1}^{0} & =Z_{11} \tilde{B}+Z_{12} \tilde{W}_{3}+Z_{13} \tilde{H}_{d}+Z_{14} \tilde{H}_{u}, \\
\tilde{t}_{1} & =\tilde{t}_{L} \cos \theta_{\tilde{t}}+\tilde{t}_{R} \sin \theta_{\tilde{t}},
\end{aligned}
$$

where $Z_{i j}$ are the mixing elements in the neutralino sector and $\theta_{\tilde{t}}$ is the mixing angle for $\tilde{t}$. Polarization of $t$ originating from the $\tilde{t}_{1}$ decay depends on the composition (which determine the coupling) of the $\tilde{t}_{1}$ and $\tilde{\chi}_{1}^{0}$, gaugino components preserve the polarization and Higgsino components flip it, this is summarized in Tab. 1. 
Table 1: Chirality of top quark from $\tilde{t_{1}}$ decay [Eq. 4.1] for different compositions of neutralino and top squark states.

\begin{tabular}{|c|c|c|}
\hline$\tilde{\chi}_{1}^{0}$ & $\tilde{t}_{1}$ & $t$ chirality \\
\hline Bino like or Wino Like & $\tilde{t}_{L}$ & $t_{L}$ \\
& $\tilde{t}_{R}$ & $t_{R}$ \\
\hline Higgsino like & $\tilde{t}_{L}$ & $t_{R}$ \\
& $\tilde{t}_{R}$ & $t_{L}$ \\
\hline
\end{tabular}

For our study, we select the final state containing one isolated lepton (either electron or muon) and at least one $b$-jet with large amount $(30 \mathrm{GeV})$ of missing energy due to the presence of weakly interacting neutralinos. The relevant parameters for the event are set as:

$$
m_{\tilde{\chi}_{1}^{0}}=100 \mathrm{GeV} ; \quad m_{\tilde{t}_{1}}=1 \mathrm{TeV} ; \quad \tan \beta=10 .
$$

The impact of our observables are presented in Fig. 4.

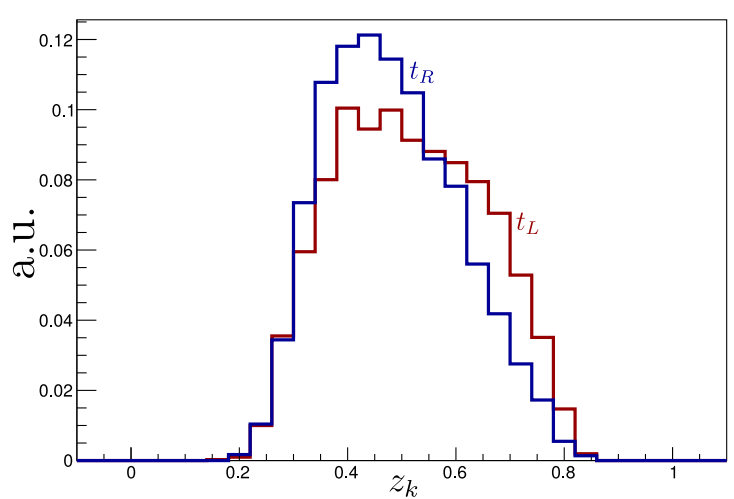

The energy fraction $z_{k}$ from Ref. [8]

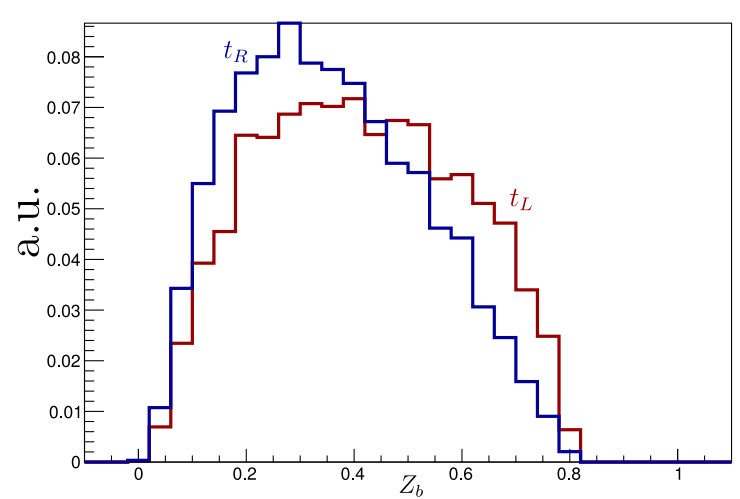

Energy fraction of $b$-like subjet $\left(E_{b} / E_{t}\right)$

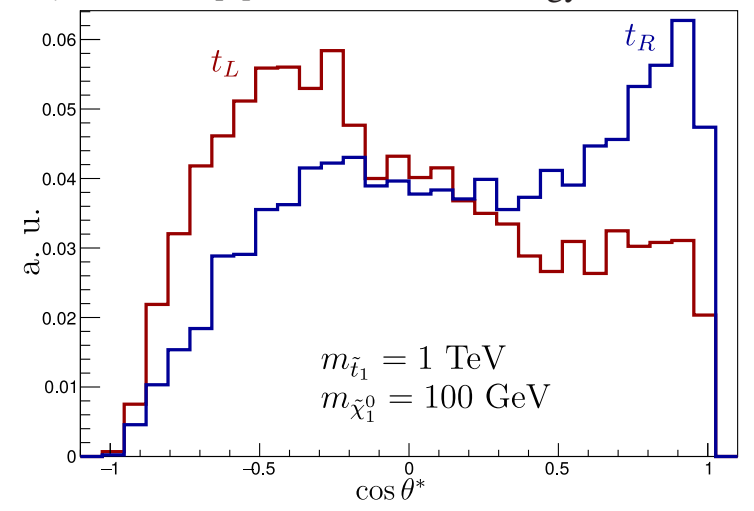

The $\cos \theta^{*}$ distribution (Eq. 2.2)

Figure 4: Distributions of the various observables for left (red) and right (blue) handed top quark for the benchmark process Eq. 4.1 where $\tilde{\chi}_{1}^{0}$ is pure bino and with the parameters given by Eq. 4.2.

We next proceed to analyze the asymmetry as a function of model parameters in various scenario, these studies are presented in Fig. 5 and Fig. 6. 


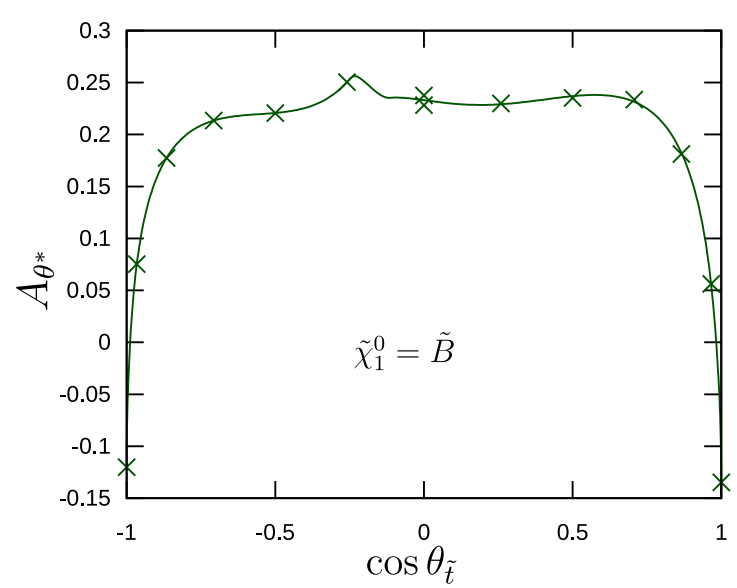

Pure Bino like $\tilde{\chi}_{1}^{0}$.

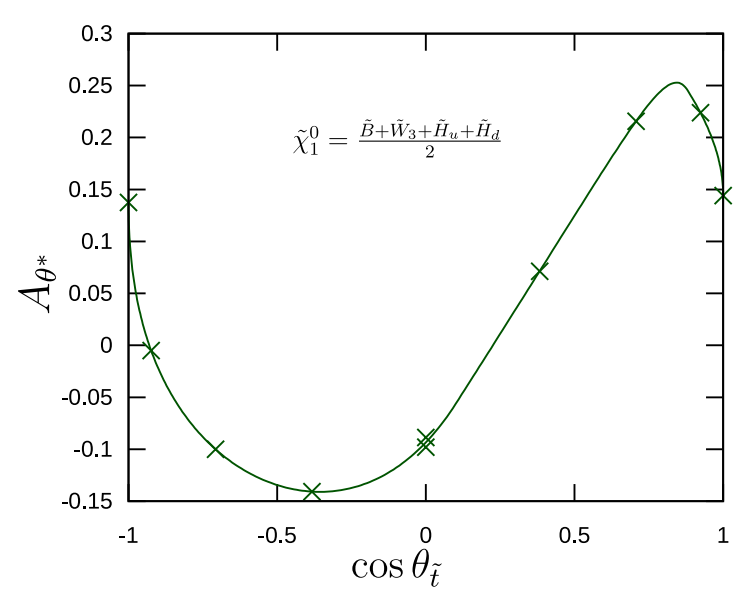

Equally mixed $\tilde{\chi}_{1}^{0}$.

Figure 5: Variation of asymmetry (Eq. 3.3) with the composition of $\tilde{t}_{1}$.

In Fig. 5, we fix the composition of $\tilde{\chi}_{1}^{0}$ and study the asymmetry with variation of $\theta_{\tilde{t}}$. When $\tilde{\chi}_{1}^{0}$ is pure bino like, the asymmetry trend mostly follows the expectation where $\theta_{\tilde{t}} \approx 0$ would lead to right handed $\tilde{t}_{1}$ decaying to right handed $t$ and hence $A_{\theta^{*}}>0$. On the other hand for $\theta_{\tilde{t}} \sim 1$ or -1 , $t$ will be left polarized and hence asymmetry is negative. The slope of the curve is explained by the fact that hypercharge of $\tilde{t}_{R}$ is twice that of $\tilde{t}_{L}$. Analyzing the mixed LSP is more challenging, but the broad features are predicted by the fact that Higgsino couplings are dominant over gaugino couplings.

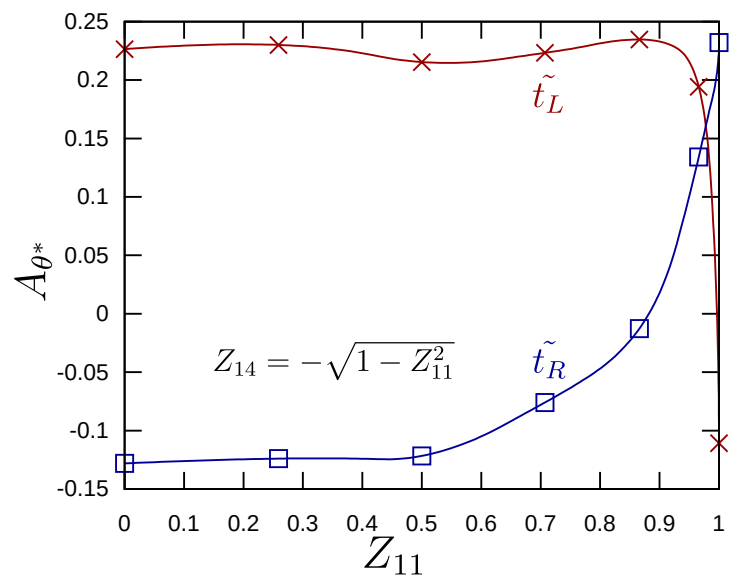

Variation among bino-Higgsino.

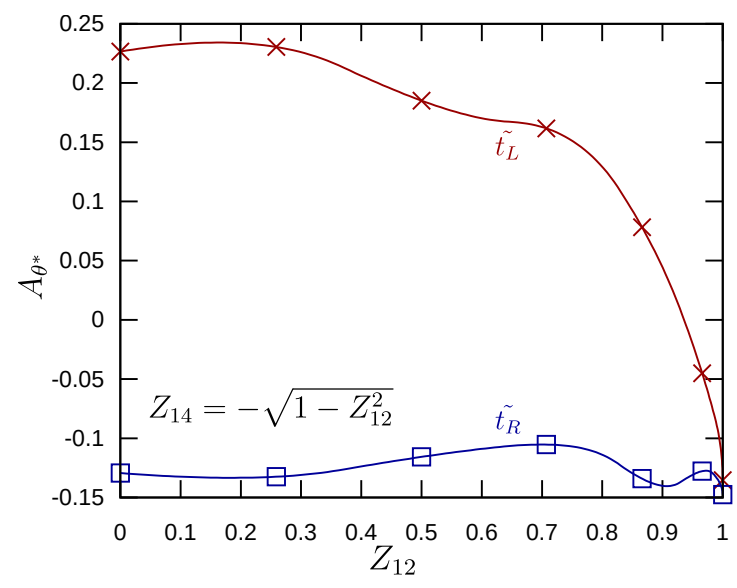

Variation among wino-Higgsino.

Figure 6: Variation of asymmetry (Eq. 3.3) with the composition of Bino or wino like with Higgsino like $\tilde{\chi}_{1}^{0}$.

In Fig. 6, we fix the composition of $\tilde{t}_{1}$ as either pure $\tilde{t}_{L}$ or pure $\tilde{t}_{R}$ while varying the composition of $\tilde{\chi}_{1}^{0}$ among Higgsino and either wino or bino components. We again see that the asymmetry is flipped when $Z_{11}$ or $Z_{12}$ is 0 (as Higgsino component is dominant), the trend for left side in Fig. 6 
holds no surprises. $\tilde{t}_{R}$ doesnot couple to wino and hence the curve for $\tilde{t}_{R}$ on the right side of Fig. 6 is essentially flat while the curve for $\tilde{t}_{L}$ varies as expected.

\section{Conclusion}

We have explored a few observables to measure the polarization of boosted hadronically decaying tops and presented a new very powerful observable which was then used to evaluate event asymmetries. The measurement of asymmetry is found to be a robust tool to probe the polarization of top quark, and the nature of couplings involved in the process. For instance, if $\tilde{t}_{1}$ is discovered, and its polarization is measured, then it will allow us to constrain it's compositions with respect to the composition of $\tilde{\chi}_{1}^{0}$. We have not performed a detailed analysis of the robustness of the observables under the effect of various selection cuts used in a realistic analysis to remove background and contamination, this exercise is left for a future study.

\section{References}

[1] R. Godbole, M. Guchait, C. K. Khosa, J. Lahiri, S. Sharma, and A. H. Vijay, "Boosted top quark polarization," Phys. Rev. D 100 (Sep, 2019) 056010, arXiv:1902.08096 [hep-ph]. https://link.aps.org/doi/10.1103/PhysRevD.100.056010.

[2] T. Plehn and M. Spannowsky, “Top Tagging,” J. Phys. G39 (2012) 083001, arXiv: 1112.4441 [hep-ph].

[3] J. M. Butterworth, A. R. Davison, M. Rubin, and G. P. Salam, "Jet substructure as a new Higgs search channel at the LHC," Phys. Rev. Lett. 100 (2008) 242001, arXiv:0802.2470 [hep-ph] .

[4] A. Brandenburg, Z. G. Si, and P. Uwer, "QCD corrected spin analyzing power of jets in decays of polarized top quarks," Phys. Lett. B539 (2002) 235-241, arXiv: hep-ph/0205023 [hep-ph].

[5] Z. Sullivan, "Fully Differential $W^{\prime}$ Production and Decay at Next-to-Leading Order in QCD," Phys. Rev. D66 (2002) 075011, arXiv: hep-ph/0207290 [hep-ph] .

[6] D. Duffty and Z. Sullivan, "Model independent reach for W-prime bosons at the LHC," Phys. Rev. D86 (2012) 075018, arXiv:1208.4858 [hep-ph].

[7] T. Sjöstrand, S. Ask, J. R. Christiansen, R. Corke, N. Desai, P. Ilten, S. Mrenna, S. Prestel, C. O. Rasmussen, and P. Z. Skands, “An Introduction to PYTHIA 8.2,” Comput. Phys. Commun. 191 (2015) 159-177, arXiv:1410.3012 [hep-ph].

[8] D. Krohn, J. Shelton, and L.-T. Wang, "Measuring the Polarization of Boosted Hadronic Tops," JHEP 07 (2010) 041, arXiv:0909.3855 [hep-ph]. 